\title{
Dimethyl Fumarate Ameliorates Lipopolysaccharide- induced Acute Lung Injury by Inhibiting NLRP3 Inflammasome-mediated Pyroptosis
}

Huayu Li

Guangdong Pharmaceutical University

Mengyan Li

Guangdong Pharmaceutical University

Chao Dong

Guangdong Pharmaceutical University

Bing Liu ( $\square$ liubing520@gdpu.edu.cn )

Guangdong Pharmaceutical University https://orcid.org/0000-0002-3144-5673

\section{Research Article}

Keywords: Acute lung injury, Dimethyl fumarate, NLRP3 inflammasome, Pyroptosis, Nrf2

Posted Date: June 25th, 2021

DOI: https://doi.org/10.21203/rs.3.rs-626638/v1

License: (c) (i) This work is licensed under a Creative Commons Attribution 4.0 International License.

Read Full License 


\section{Abstract}

Acute lung injury (ALI) and acute respiratory distress syndrome (ARDS) are clinically severe respiratory disorders, and there are currently no Food and Drug Administration-approved drug therapies. It is established that Dimethyl fumarate (DMF) exhibits anti inflammatory effects, however, the specific effect of DMF on ALI remains largely unknown. The aim of the present study was to investigate whether, and by which mechanism, DMF alleviated lipopolysaccharide (LPS)-induced ALI. We found that intraperitoneal injection of DMF markedly reduced the pulmonary injury, decreased pulmonary edema and pulmonary permeability. Emerging studies suggested that the NOD-like receptor pyrin domain containing 3 (NLRP3) inflammasome-mediated pyroptosis played a critical role during ALI. NLRP3 inflammasome-mediated pyroptosis is significantly activated with the cleavage of caspase- 1 and GSDMD occurring in the lung of LPS-induced ALI. DMF inhibited the activation of the NLRP3 inflammasome and pyroptosis in both lung of ALI mice and LPS-induced BEAS-2B cells. Mechanistically, DMF enhanced expressions of Nuclear factor erythroid-2-related factor 2 (Nrf2), leading to inactivation of NLRP3 inflammasome and reduction of pyroptosis in both ALI mice and LPS-induced BEAS-2B cells. Conversely, Nrf2 inhibitor reduced the inhibitory effects of DMF on NLRP3 inflammasome and pyroptosis, and consequently blocked the improvement roles of DMF on ALI in mice. This study for the first time demonstrated that DMF could improve LPS-induced ALI via inhibiting NLRP3 inflammasome and pyroptosis, and that these effects were mediated by triggering Nrf2 expression, suggesting a therapeutic potential of DMF as an antiinflammatory agent for ALI/ARDS treatment.

\section{Introduction}

Acute lung injury (ALI) and its more severe form, acute respiratory distress syndrome (ARDS) is a critical pathological condition especially in some severe infectious respiratory diseases, it is characterized by alveolar-capillary membrane disruption, extensive leukocyte infiltration and release of pro-inflammatory mediators, pulmonary edema associated with proteinaceous alveolar exudates and deterioration of gas exchange, and finally respiratory failure [1]. Despite recent advances in clinical management and extensive investigations in new strategies for treatment, there is still no Food and Drug Administration (FDA)-approved treatment for ALI/ARDS [2]. Endotoxin or lipopolysaccharide (LPS) derived from Gramnegative bacteria has been well recognized in the pathogenesis of ALI. In vivo intratracheal administration of LPS has been extensively used as a clinically relevant model [3].

The Nod-like receptor protein 3 (NLRP3) inflammasome is a multi-protein complex of the innate immune system, consisting of NLRP3, the adaptor protein apoptosis-associated speck-like protein containing a caspase recruitment domain (ASC) and inflammatory caspase-1 [4]. Activation of the NLRP3 inflammasome requires two signals: a priming event is mediated by microbial molecules or endogenous cytokines, which upregulate the expression of NLRP3 and precursors of IL-1 $\beta$ (pro-IL-1 $\beta$ ) through activating the nuclear factor-KB (NF-KB) signaling pathway, and a subsequent assembly of NLRP3 with ASC and precursors of caspase-1 (pro-caspase-1) [5, 6]. NLRP3 inflammasome assembly triggers the activation of inflammatory caspase- 1 and process of pro-inflammatory cytokines such as IL-1ß. In 
addition, caspase- 1 can cleave gasdermin D (GSDMD) to yield an N-terminal cleavage product (GSDMDNT), which induces pyroptosis by forming plasma membrane pores [7]. The hallmark characteristics of pyroptosis are pore formation, osmotic swelling and early loss of membrane integrity [8]. The inflammasome/caspase-1 pathway promotes the development of ALI through the production of interleukin(IL)-1 $\beta$ and IL-18, and caspase-1 activation leads to lung injury and pulmonary inflammation [9]. Conversely, the caspase-1 inhibitor Ac-YVAD-CMK could restrain Inflammatory aggregation and pulmonary edema in LPS-induced ALI [10]. Therefore, inhibiting NLRP3 inflammasome and pyroptosis to suppress the excessive inflammatory response may develop a novel therapeutic strategy for ALI.

Owing to the immunomodulatory and anti-inflammatory effects, Dimethyl fumarate (DMF) has been approved by FDA with a good safety record in both psoriasis and multiple sclerosis [11]. Recently, several studies have shown that DMF elicited favorable effects on other type diseases. For example, Yang Yao et al. reported that DMF reduced infarct size during the subacute stage of stroke, and their protective role was likely mediated by Nuclear factor erythroid-2-related factor 2 (Nrf2) pathway [12]. DMF lessened vitamin D3-induced vascular calcification in mice [13]. Importantly, it was shown that DMF inhibited NLRP3 inflammasome activation in colitis, thioacetamide-induced liver and diabetic aortas [14-16]. However, whether DMF can improve ALI by inhibiting NLRP3 inflammasome and pyroptosis still need to be investigated. In current study, DMF is used for treating LPS-induced ALI in mice, and employed for LPS-induced pulmonary epithelial cells. We investigate the therapeutic effect and discover the related mechanisms in ALI.

\section{Materials And Methods}

\section{Materials}

DMF (DMF, purity > 98\%), LPS (Escherichia coli 055:B5), Dexamethasone (DXMS) was purchased from Med Chem Express Co. (SH, USA). Brusatol (BT, Nrf2 inhibitor) was purchased from Tauto biotech (SH, China). Enzyme-linked immunosorbent assay (ELISA) kits for IL-1 $\beta$ and IL-18 was purchased from MultiSciences (Lianke) Biotech Co., Ltd. (HZ, China). Active caspase-1 was determined using the FAMYVAD-FMK caspase-1 detection kit (Cell technology, Mountain View, CA, USA) according to the manufacturer's protocol. 2,7-Dichlorofluorescein diacetate (DCFH-DA) was purchased from Sigma-Aldrich (St. Louis, MO, USA). Antibodies against Nrf2, HO-1, NQ01, NF-KB, Txnip, NLRP3, casapase-1, IL-1 $\beta$, GSDMD, and $\beta$-Tubulin were supplied by ABclonal Technology Co.,Ltd. (WH, China), unless otherwise stated.

\section{Animals}

Five-week-old male C57BL/6 mice were purchased from Hunan SJA Laboratory Animal Co.,Ltd (Certificate SCXK2019-0004; HN, China). Mice were acclimatized to the animal facilities for at least a week before beginning experiments. All mice were kept in certified specific pathogen-free facilities maintained around $24^{\circ} \mathrm{C}$ with a $12 \mathrm{~h}$ light/dark cycle and free access to food and water. At the end of the experiment, mice were sacrificed, lung tissues and blood samples were collected. Animal handling and 
procedures were approved by the Guangdong Pharmaceutical University Health Science Center Institutional Animal Care and Use Committee (gdpulacspf2017335). The investigation conforms to the Guide for the Care and Use of Laboratory Animals published by the US National Institutes of Health (NIH Publication No. 85 - 23, revised 1996).

\section{Experimental Protocol}

To explore the therapeutic effect of DMF on ALI, C57BL/ 6 mice were randomly divided into six groups, five in each group: Control, LPS only (10 mg/kg), DMF (10 or 30 or $90 \mathrm{mg} / \mathrm{kg}$ ) + LPS, and DXMS (as a positive drug, $50 \mu \mathrm{g} / \mathrm{kg}$ ) + LPS. The mice were fed adaptively for one week and fasted and abstained from water for $12 \mathrm{~h}$ before modeling. LPS was administered intratracheally $(10 \mathrm{mg} / \mathrm{kg})$ using a $30 \mathrm{G}$ needle in mice. Equal volume of saline was administered in controls. After $12 \mathrm{~h}$, DMF $(10,30 \mathrm{or} 90 \mathrm{mg} / \mathrm{kg})$ and DXMS $(50 \mu \mathrm{g} / \mathrm{kg})$ were administered intraperitoneally. After DMF and DXMS administration for $12 \mathrm{~h}$, the animals were euthanized by $\mathrm{CO}_{2}$ asphyxiation. Subsequently, lung tissue samples and bronchoalveolar lavage fluid (BALF) were collected and used for Hematoxylin and eosin (H\&E) staining, Western blot and ELISA assay.

To explore the therapeutic mechanism of DMF on ALI, C57BL/6 mice were randomly divided into four groups, five in each group: Control, LPS only $(10 \mathrm{mg} / \mathrm{kg})$, DMF $(90 \mathrm{mg} / \mathrm{kg})+\mathrm{LPS}$, and BT $(2 \mathrm{mg} / \mathrm{kg})+$ DMF $(90 \mathrm{mg} / \mathrm{kg})+$ LPS. The mice were fed adaptively for one week and fasted and abstained from water for $12 \mathrm{~h}$ before modeling. LPS was administered intratracheally $(10 \mathrm{mg} / \mathrm{kg})$ using a $30 \mathrm{G}$ needle in mice. Equal volume of saline was administered in controls. BT $(2 \mathrm{mg} / \mathrm{kg})$ was pre-injected. The rest are described above.

\section{Histopathological Evaluation}

The left lungs of mice were excised at $24 \mathrm{~h}$ after the LPS challenge. A histopathological examination was performed on the mice that were not subjected to BALF collection. The lung tissue samples were immersed in normal $10 \%$ neutral buffered formalin and fixed for $48 \mathrm{~h}$, dehydrated in a series of graded ethanol, embedded in paraffin wax, and cut into 5 - $\mu$ m-thick sections. The paraffin-embedded sections were stained with H\&E for pathological analysis. Images were acquired on ZEISS microscope. The scores of lung injury were blindly evaluated. Each histological characteristic was scored from 0 (normal) to 5 (maximal) according to the sum of the score for damage level such as the number of infiltration cells, alveolar wall thickening, patchy hemorrhage and interstitial edema [17].

\section{Lung Wet/Dry (W/D) Ratios}

Lung samples were collected $24 \mathrm{~h}$ after LPS stimulation, blotted dry and weighed immediately after removal ('wet' weight) before being subjected to desiccation in an oven at $80^{\circ} \mathrm{C}$ for $48 \mathrm{~h}$ to obtain the 'dry' weight. The ratio of wet lung weight to dry lung weight was measured by assessment of tissue edema.

\section{Protein Concentration Assay in BALF}


After LPS challenge for $24 \mathrm{~h}$, all mice were euthanized for collecting their BALF. BALF was harvested via injection and retraction of $0.5 \mathrm{~mL} 1 \times$ PBS three times. The collected BALF was then centrifuged, and then their protein concentrations were directly measured using BCA protein assay kit.

\section{ELISA Assay}

The lung tissue was obtained from each sample in vivo, Tissue homogenated, centrifuged, collected supernatants for measurement of the IL-1 $\beta$ and IL-18 secretion using an ELISA kit as the manufacturer's instructions, respectively. In addition, BEAS-2B cells were grown in 24-well plates $\left(2 \times 10^{4}\right.$ cells/well) for 12 h. Subsequently, cells were subjected to different concentrations of the drug, the cell-free supernatants were collected for analysis of the IL-1 $\beta$ secretion. The optical density from each well was detected at 450 $\mathrm{nm}$ and $570 \mathrm{~nm}$.

\section{Western Blotting}

Lung tissue samples or cells were lysed in a RIPA buffer with protease and phosphatase inhibitors for 30 min. The protein concentrations were measured using a BCA protein assay kit, and $30 \mu \mathrm{g}$ of proteins were electrophoretically transferred onto a PVDF membrane following separation on a $10 \%$ SDS-

polyacrylamide gel. The membrane was blocked with blocking solution $(5 \%(\mathrm{w} / \mathrm{v})$ nonfat dry milk) for $1 \mathrm{~h}$, followed by an overnight incubation at $4{ }^{\circ} \mathrm{C}$ with a specific primary antibody. The following day, the membrane was incubated for an additional $1 \mathrm{~h}$ with HRP-conjugated secondary antibody (1:5000 dilution) at room temperature after thoroughly washing three times with TBST. Bands were detected by $\mathrm{ECL}$ and band intensities were quantified using Image $\mathrm{J}$ gel analysis software.

\section{Cell Culture and MTT Assay}

The human pulmonary epithelial cells (BEAS-2B cells) was purchased from American Type Culture Collection (ATCC). BEAS-2B cells were cultured in Dulbecco's Modified Eagle Medium (DMEM) containing $10 \% \mathrm{FBS}$ in incubator with $5 \% \mathrm{CO}_{2}$ and humidified air at $37^{\circ} \mathrm{C}$. Cell viability was measured by an MTT assay in accordance with the manufacturer's instructions. BEAS-2B cells were seeded into 96-well plates $\left(3 \times 10^{3}\right.$ cells/well) for $24 \mathrm{~h}$ and exposed to LPS $(10 \mu \mathrm{g} / \mathrm{mL})$, and then treated with different concentrations of DMF (final concentration: $0,2.5,5,10 \mu \mathrm{M})$ for $24 \mathrm{~h}$. Subsequently, MTT $(5 \mathrm{mg} / \mathrm{mL}$ ) was added to the cells, which were then incubated for an additional $4 \mathrm{~h}$. DMSO was added to dissolve the formazan crystals, and the absorbance was measured at $570 \mathrm{~nm}$. The cell viability was then determined with the following equation: Cell viability $(\%)=$ (mean of Abs. of treatment group/mean Abs. of control) $\times 100 \%$. The other drug treatment groups were treated with final concentrations of DXMS $(100 \mathrm{nM})$ and BT $(300 \mathrm{nM})$.

\section{Flow Cytometry}

Active caspase-1 was determined using the FAM-YVAD-FMK caspase-1 detection kit. Cell death stain (7$A A D)$, a fluorescent nucleic acid dye which only penetrates ruptured cell membranes, was used to mark cells with membrane pore formation. Briefly, after treatment, the cells were harvested and incubated with caspase- 1 detection probe for $60 \mathrm{~min}$ at $37^{\circ} \mathrm{C}$ in the dark. At the end of incubation, the unbound reagent 
was washed away using cellular wash buffer. Cells were then stained with $1 \mathrm{mM}$ 7-AAD for a further 10 min at $37^{\circ} \mathrm{C}$ protecting from light. The cells were then analyzed using a flow cytometer.

\section{Measurement of ROS Production}

For intracellular ROS measurement, BEAS-2B cells were seeded into 6-well plates ( $2 \times 10^{5}$ cells/well) for 24 $\mathrm{h}$ incubation, and then recovered in serum-free DMEM. Subsequently, cells were subjected to LPS and DMF for additional $24 \mathrm{~h}$ and washed with PBS twice for DCFH-DA staining.

\section{Real-time RT-PCR}

Total RNA was extracted from cells using Trizol Reagent (Invitrogen), and then complementary DNA (cDNA) was synthesized using ReverTra Ace reverse transcriptase (TOYOBO, Japan, FSQ-301) according to the manufacturer's instructions. Real-time RT-PCR was performed with the SYBR Green Realtime PCR Master Mix (TOYOBO, Japan, QPK-201) on an iCycler (Bio-Rad) following the manufacturer's instructions. The primers used in real-time quantitative PCR. The primer sequences were as follows: Nrf2 forward primer: 5'-GACGTGTGGCGGCTGAGC-3'; Nrf2 reverse primer: 5'-GCACCGCGTCCGAACTAGAAG-3'; HO-1 forward primer: 5'- GGTGCTCGTACTGCTACTGTCATG-3'; HO-1 reverse primer: 5'GCCACGAACCTCATCTCTTCCAC-3'; NQ01 forward primer: 5'- CGCCTGCCATCATGCCTGAC-3'; NQ01 reverse primer: 5'- GTGTGGTGGATCACGCCTGTAATC-3'; GAPDH forward primer: 5'GGCACCGTCAAGGCTGAGAAC-3'; GAPDH reverse primer: 5'- CATGGTGGTGAAGACGCCAGTG-3'. The gene expression levels for each amplicon were calculated using the ${ }^{\triangle \Delta} \mathrm{CT}$ method and normalized against GAPDH mRNA.

\section{Statistical Analysis}

All data referenced above were expressed as the means \pm SD (Standard Deviation). All of the graphs were generated with GraphPad Prism 7.0. (GraphPad Software Inc., La Jolla, CA, USA). For comparative studies, t-test (unpaired) and one-way ANOVA followed by Dunnett's post hoc tests were used respectively. $P$ values $<0.05$ were considered significant.

\section{Result}

\section{DMF Improved LPS-Induced ALI and Inhibited NLRP3 Inflammasome and Pyroptosis in ALI MICE}

To evaluate the effect of DMF on ALI, we first used LPS to conduct ALI mice model. H\&E staining and lung injury score system were utilized to assess the pathological changes of the lung tissues in this study. As shown in Fig. 1a-c, there were no pathological changes in the lung tissue of control group. In contrast, in the lung tissues of LPS group mice, notable the number of infiltration cells, alveolar wall thickening, patchy hemorrhage and interstitial edema were observed. Treatment with different concentration of DMF markedly alleviated LPS-induced these pathological changes of lung, and DXMS exhibited a similar effect with $90 \mathrm{mg} / \mathrm{kg}$ DMF treatment. In addition, non-cardiogenic pulmonary edema 
and protein leakage in BALF were critical features of ALI [18]. Lung wet to dry weight (W/D) ratio was obtained to evaluate the severity of pulmonary edema. As showed in Fig. 1d, e, LPS injection markedly increased lung injury scores, W/D ratio and protein leakage in BALF, which were all significantly attenuated by DMF or DXMS intervention. As showed in Fig. 1f, LPS alone significantly increased the serum levels of IL-1 $\beta$ and IL-18, while DMF treatment significantly inhibited this process in ALI mice.

We further found that LPS-induced ALI were associated to NLRP3 inflammasome activation and pyroptosis, which were evidenced by increased expression of NLRP3, cleavage of caspase-1, IL-1 $\beta$ and GSDMD in the lung tissues (Fig. 1g). LPS treatment also upregulated priming signal NF-KB and Thioredoxin binding protein (Txnip) expressions (Fig. 1g). Next, we further investigated whether DMF could restrain NLRP3 inflammasome activation and pyroptosis in ALI mice. Results showed that DMF suppressed NLRP3 inflammasome activation and pyroptosis while increased Nrf2 expression, which consequently alleviated lung injury in ALI mice (Fig. 1g).

These results suggested DMF improved LPS-induced ALI by inactivating NLRP3 inflammation and pyroptosis.

\section{Nrf2 Inhibitor Reduced the Beneficial Effects of DMF in LPS- Induced ALI MICE}

To further validate the mechanistic underlying DMF-mediated beneficial effects on LPS-induced ALI and to validate if the observed effects are mediated through Nrf2 signaling. BT was pre-injected into mice to inhibit Nrf2 activity in ALI mice. As shown in Fig. 2a-d, LPS-induced lung pathological changes could be significantly attenuated by DMF administration, and lung injury scores, W/D ratio and protein leakage in BALF were restored by DMF administration, notably, the beneficial effects were suppressed by BT preadministration. Meanwhile, as shown in Fig. 2e, hyperactivation of NLRP3 inflammasome and pyroptosis were inhibited by DMF administration in ALI mice, and these inhibitory effects were all reduced by BT.

These results indicated that DMF could improve LPS-induced ALI via inhibiting NLRP3 inflammasome and pyroptosis, and that these effects were at least partly mediated by triggering Nrf2 expression.

\section{DMF Inhibited LPS-Induced NLRP3 Inflammasome Activation and Pyroptosis in Pulmonary Epithelial Cells (BEAS-2B Cells)}

LPS-stimulated pulmonary epithelial cells were used to establish in vitro model of ALI [19]. Based on in vivo observation, we further evaluated the effect of DMF on BEAS-2B cells. Firstly, the effect of DMF on cell viability was measured by an thiazolyl blue tetrazolium bromide (MTT) assay in BEAS-2B cells. As shown in Fig. 3a, LPS markedly decreased the viability of BEAS-2B cells in a dose-dependent manner. In addition, DMF slightly affected cell growth in a dose-dependent manner with the half-maximal inhibitory concentration $\left(\mathrm{IC}_{50}\right)$ of $44.23 \pm 0.15 \mu \mathrm{M}$ (Fig. 3b). To avoid the acute cytotoxicity, the usage of DMF at a 
concentration of $0-10 \mu \mathrm{M}$ was chosen for the best experimental condition. To investigate the role of DMF on LPS-induced cytotoxicity in BEAS-2B cells, cells were treated with different concentrations of DMF. As shown in Fig. 3c, DMF dose-dependently rescued LPS-induced cell cytotoxicity in BEAS-2B cells. Moreover, LPS significantly increased production of IL-1 $\beta$, while DMF treatment considerably inhibited LPS-induced IL-1 $\beta$ generation in the supernatant of BEAS-2B cells (Fig. 3d).

During pyroptosis, GSDMD-NT can combine with lipid in the plasma membrane and form large oligomeric pores, leading to the positive staining of dead cells, which can be determined by 7-AAD staining [20]. Contrary to the LPS alone treatment cells, in which include more population of active caspase- $1^{+} / 7-\mathrm{AAD}^{+}$cells, and more cleavage of caspase- 1 and GSDMD, DMF efficiently interrupted all these events in BEAS-2B cells, suggesting that DMF could reduce LPS-induced cell pyroptosis (Fig. $3 e, f)$. Moreover, the results shown that LPS-induced NLRP3 inflammasome was significantly blunted by DMF, as evidenced by the reduction in Txnip and NLRP3 expression, and cleavage of caspase-1, IL-1 $\beta$ (Fig. 3f). It's worth noting that LPS induced priming of NLRP3 inflammasomes also reduced by DMF, as evidenced by the decreased expression of NF-KB in BEAS-2B cells (Fig. 3f).

All these results suggested that DMF inhibit LPS-induced NLRP3 inflammasome activation and pyroptosis in pulmonary epithelial cells.

\section{DMF Mitigated ROS Generation and Promoted Nrf2 Expression in LPS-Stimulated BEAS-2B Cells}

Due to oxidative damage plays a key role in LPS-induced ALI, we examined whether DMF treatment could inhibit LPS-triggered reactive oxygen species (ROS) overgeneration. Indeed, as shown in Fig. 4a, LPS stimulation led to significantly increased intracellular ROS contents, while DMF treatments circumvented the elevation of ROS levels, implicating that DMF could reduce ROS generation in BEAS-2B cells. To explore the possible mechanism involved in the antioxidant effect of DMF, the activation of Nrf2 signaling pathway was examined since Nrf2 is well-known to trigger the transcription of proteins such as HO-1 and Dehydrogenase, quinone 1 (NQO1), in antioxidant defense system under oxidative stress. As shown in Fig. 4b, c, DMF counteracted the suppression of Nrf2, HO-1 and NQO1 induced by LPS at mRNA and protein level in BEAS-2B cells. These results demonstrated that DMF could attenuate ROS generation and enhance Nrf2 antioxidant signals in LPS-induced BEAS-2B cells.

\section{DMF Decreased NLRP3 Inflammasome-Mediated Pyroptosis by Promoting Nrf2 Expression in BEAS-2B Cells}

To ascertain whether the protective effect of DMF against LPS-induced ALI is mediated by Nrf2 activation, BT was used to specifically inhibit Nrf2 activity in BEAS-2B cells. As shown in Fig. 5a-c, ROS generation, expressions of Txnip and NLRP3, cleavage of caspase- 1 and IL-1 $\beta$, as well as population of active caspase $1^{+} / 7-A A D^{+}$cells were remarkably increased in BEAS-2B cells treated with LPS, however, DMF failed to suppress all these events in cells pretreatment with BT. These results demonstrated that 
DMF could attenuate LPS-induced NLRP3 inflammasome activation and related pyroptosis by upregulating Nrf2 expression in BEAS-2B cells.

\section{Discussion}

In this study, we aimed to explore the therapeutic potential of DMF against LPS-induced ALI. Despite its well-documented anti-inflammatory and antioxidant properties, DMF has not yet been studied in experimental models of LPS-induced ALI. Our results indicated that DMF may attenuate LPS-induced ALI in terms of improved lung inflammatory and reduced pyroptosis due in part to preserve NLRP3 inflammasome activation via Nrf2-mediated signaling (Fig. 6).

Accumulating evidences show that multiple pathological processes, such as capillary permeability increase, extensive neutrophil infiltration, inflammatory mediators release and edema, are displayed in the pathogenesis of ALI [21, 22]. In accordance with previous studies, our data showed that LPS could induce pulmonary edema, coagulation and inflammation cells, reduced neutrophil numbers. In our study, we found that the elevation of lung W/D ratios and protein level in BALF accounting for an increased pulmonary permeability and lung edema in LPS-instillation lungs, were markedly also attenuated by DMF treatment. Our study strongly suggested that DMF could improve LPS-induced ALI in mice. Clinical evidences demonstrated the superiority of DMF in treating multiple sclerosis patients and preclinical studies have shown that DMF could treat several human diseases including lung disease. CattaniCavalieri et al. found that DMF could attenuate lung injury induced by chronic exposure to diesel exhaust particles in mice [23]. Grzegorzewska et al. reported that DMF ameliorates pulmonary arterial hypertension and bleomycin-induced lung fibrosis [11]. In combination of these results, our studies indicated that DMF may be a promised drug protecting from a variety of factors-induced lung injury not limiting to LPS.

NLRP3 inflammasome play an important role in the pathogenesis of ALI. Wu et al. reported that NLRP3 inflammasome of alveolar macrophage is associated with ALI/ARDS in mice [24]. Furthermore, Zhang et al. found that NLRP3 inflammasome contributed to LPS-induced ALI while NLRP3 deficiency or inhibition attenuated lung histopathological injury, inflammatory cell infiltration [17]. We found that NLRP3 inflammasome was markedly activated in lung tissue of ALI mice and the plasma level of IL-1 $\beta$ was significantly increased in ALI mice, which were consistent with previous studies [24]. Emerging evidences showed DMF inhibited NLRP3 inflammasome activation in colitis, thioacetamide-induced liver and diabetic aortas [14-16]. In line with these findings, we found that the administration of DMF significantly inhibited NLRP3 inflammasome activation in lung tissue of ALI, as evidenced by decreased expression of NLRP3, cleavage of caspase- 1 and IL-1 $\beta$. These results indicated that the protective effect of DMF on LPS-induced ALI was associated with the inhibition of NLRP3 inflammasome. Interestingly, DMF has also inhibited NF-KB expression both in LPS-induced ALI mice and pulmonary epithelial cells, suggesting that inhibiting both "priming" and "assembly" signals was related to the protective effect of DMF on LPSinduced ALI. 
The assembly of NLRP3 inflammasome triggers the autocleavage of pro-caspase- 1 into active caspase1[25]. Followed NLRP3 inflammasome activation, caspase-1 cleaves GSDMD to yield GSDMD-NT, which critically determines the fate of pyroptotic cell death [26]. Driven by inflammasome activation, pyroptotic cell death in pulmonary epithelial cells exacerbated lung damage in ALI [27]. Consistent with previous reports, we found that LPS promoted the cleavage of GSDMD in lung tissue of ALI, indicating the presence of pyroptosis in the ALI.

Pyroptosis leads to the formation of pores in the plasma membrane that allow a membrane-impermeant dye like 7-AAD to enter pyroptotic cells but not apoptotic or viable cells [20]. Considering that pulmonary epithelial cells have a profound impact on the development and prognosis of ALI by releasing inflammatory cytokines, BEAS-2B cells was subjected to LPS stimulation were used to seek whether DMF treatment inhibited NLRP3 inflammasome and related pyroptosis in vitro. Accumulating evidences supported that NLRP3 inflammasome pathway was activated under LPS stimulation. Correspondingly, we confirmed that LPS activated NLRP3 inflammasome and induced pyroptosis in BEAS-2B cells, as evidenced by increased cleavage of GSDMD and active caspase- $1^{+} / 7-\mathrm{AAD}^{+}$cells. Our further expriments indicated that DMF treatment not only inhibited LPS-induced the expression of NLRP3 inflammasome but also significantly reduced pyroptosis in BEAS-2B cells.

Oxidative stress created by LPS plays an important role in the pathology of LPS-induced ALI [28]. Txnip is involved in inflammation in response to oxidative stress [29]. In the resting state, Txnip is kept in an inactive form by binding with its endogenous inhibitor thioredoxin (TRX). Both Txnip and TRX regulate and maintain the balance of intracellular oxidation and anti-oxidation systems. When Txnip separates from TRX, it directly activates the NLRP3 inflammasome under oxidative stress [30]. Here we clearly presented that Txnip acted as an upstream factor regulated by LPS, and thus led to NLRP3 inflammasome activation and pyroptosis, which were all reversed by DMF.

Nrf2 is a pleiotropic transcription factor through antioxidant response elements within the regulatory region of many target genes such as $\mathrm{HO}-1$ and NQ01, coordinates antioxidative allowing all types of cells adapt to detrimental conditions caused by intracellular or extracellular stress [28, 31]. In this study, we found that DMF obviously promoted the expression of Nrf2 and inhibited the production of ROS in LPSinduced BEAS-2B cells. We further inhibited Nrf2 activity to confirm seek whether DMF inhibited NLRP3 inflammasome activation by upregulaying Nrf2 signaling. Our results demonstrated that inhibition of Nrf2 abrogated the protective effects of DMF on inhibiting NLRP3 inflammasome activation and related pyroptosis in LPS-induced ALI in vitro and in vivo. Taken together, DMF directly activated LPS-induced Nrf2 expression, thus leading to inactivation of NLRP3 inflammasome and inhibition of pyroptosis.

Collectively, the results we presented here revealed that DMF exerts therapeutic effects in LPS-induced ALI by inhibiting NLRP3 inflammasome activation and related pyroptosis. Moreover, we uncovered its therapeutic effect at least partially through promoting Nrf2 expression. Our study identified the benefit of DMF for ALI and provided more evidences for therapeutical value of DMF on diseases which were associated with NLRP3 inflammasome and related pyroptosis.

Page $10 / 21$ 


\section{Conclusions}

Our data implied that DMF could improve LPS-induced ALI via inhibiting NLPR3 inflammasome and pyroptosis, and that these effects were mediated by triggering Nrf2 expression, suggesting a therapeutic potential of DMF as an anti-inflammatory agent for ALI/ARDS treatment.

\section{Abbreviations}

acute lung injury (ALI)

acute respiratory distress syndrome (ARDS)

bronchoalveolar lavage fluid (BALF)

Brusatol (BT)

2,7-Dichlorofluorescein diacetate (DCFH-DA)

Dimethyl fumarate (DMF)

Dexamethasone (DXMS)

Gasdermin D (GSDMD)

Gasdermin D N-terminal cleavage product (GSDMD-NT)

Hematoxylin and eosin (H\&E)

Heme oxygenase 1 (HO-1)

the half-maximal inhibitory concentration $\left(\mathrm{IC}_{50}\right)$

interleukin (IL)

lipopolysaccharide (LPS)

3-(4,5-dimethyl-2-thiazolyl)-2,5-diphenyl-2-H-tetrazolium bromide, Thiazolyl Blue Tetrazolium Bromide (MTT)

nuclear factor-kB (NF-kB)

Nod-like receptor protein 3 (NLRP3)

Dehydrogenase, quinone 1 (NQ01)

Nuclear factor erythroid-2-related factor 2 (Nrf2)

precursors of interleukin-1 $\beta$ (Pro IL-1 $\beta$ )

Reactive oxygen species (ROS)

thioredoxin (TRX)

Thioredoxin binding protein (Txnip) 


\section{Declarations}

\section{Ethics Approval.}

The animal experiments were approved by the Animal Care and Use Committee of Guangdong Pharmaceutical University (00242079). The investigation conforms to the Guide for the Care and Use of Laboratory Animals published by the US National Institutes of Health (NIH Publication No. 85-23, revised 1996). All authors approved the publication of the article. The data and material were available.

\section{Consent for Publication.}

Not applicable

\section{AVAILABILITY OF DATA AND MATERIALS:}

Data are available from the corresponding author on reasonable request.

\section{Competing Interests.}

The authors declare no competing interests.

\section{FUNDING:}

This work was supported by the project of the Innovation Team Projects in Universities of Guangdong Province (No. 2018KCXTD016), which was awarded by Professor Liu Bing's team.

\section{AUTHOR CONTRIBUTION}

Bing Liu designed this study, Huayu Li drafted the manuscript. Huayu Li, Mengyan Li and Chao Dong contributed equally to this work. Huayu Li, Mengyan Li and Chao Dong performed the experiments, and conducted the statistical analysis. All authors read and approved the final manuscript. All authors reviewed the results and approved the final version of the manuscript.

\section{Acknowledgements:}

The author(s) wish to thank all those who have contributed to this study.

\section{References}


1. Matthay, M., and R. Zemans. 2011. The acute respiratory distress syndrome: pathogenesis and treatment. Annual review of pathology 6: 147-163.

2. Standiford, T., and P. Ward. 2016. Therapeutic targeting of acute lung injury and acute respiratory distress syndrome. Translational research 167: 183-191.

3. Wang, H., M. Bodenstein, and K. Markstaller. 2008. Overview of the pathology of three widely used animal models of acute lung injury. European surgical research 40: 305-316.

4. Hou, L., Z. Yang, Z. Wang, X. Zhang, Y. Zhao, H. Yang, B. Zheng, W. Tian, S. Wang, Z. He, and X. Wang. 2018. NLRP3/ASC-mediated alveolar macrophage pyroptosis enhances HMGB1 secretion in acute lung injury induced by cardiopulmonary bypass. Laboratory investigation 98: 1052-1064.

5. Luo, J., F. Wang, F. Sun, T. Yue, Q. Zhou, C. Yang, S. Rong, P. Yang, F. Xiong, and Q. Yu. 2021. Targeted Inhibition of FTO Demethylase Protects Mice Against LPS-Induced Septic Shock by Suppressing NLRP3 Inflammasome. Frontiers in immunology 12: 663295.

6. dos Santos, G., M. Rogel, M. Baker, J. Troken, D. Urich, L. Morales-Nebreda, J. Sennello, M. Kutuzov, A. Sitikov, and J. Davis. 2015. Vimentin regulates activation of the NLRP3 inflammasome. Nature communications 6: 6574.

7. Zeng, C., F. Duan, J. Hu, B. Luo, B. Huang, X. Lou, X. Sun, H. Li, X. Zhang, S. Yin, and H. Tan. 2020. NLRP3 inflammasome-mediated pyroptosis contributes to the pathogenesis of non-ischemic dilated cardiomyopathy. Redox biology 34: 101523.

8. Wang, X., X. Li, S. Liu, A. Brickell, J. Zhang, Z. Wu, S. Zhou, and Z. Ding. 2020. PCSK9 regulates pyroptosis via mtDNA damage in chronic myocardial ischemia. Basic research in cardiology 115: 66.

9. Li, X., M. Xie, C. Lu, J. Mao, Y. Cao, Y. Yang, Y. Wei, X. Liu, S. Cao, and Y. Song. 2020. Design and synthesis of Leukotriene A4 hydrolase inhibitors to alleviate idiopathic pulmonary fibrosis and acute lung injury. European journal of medicinal chemistry 203: 112614.

10. Chu, Q., S. Wang, L. Jiang, Y. Jiao, X. Sun, J. Li, L. Yang, Y. Hou, N. Wang, and X. Yao. 2021. Patulin induces pyroptosis through the autophagic-inflammasomal pathway in liver. Food and chemical toxicology 147: 111867.

11. Grzegorzewska, A., F. Seta, R. Han, C. Czajka, K. Makino, L. Stawski, J. Isenberg, J. Browning, and M. Trojanowska. 2017. Dimethyl Fumarate ameliorates pulmonary arterial hypertension and lung fibrosis by targeting multiple pathways. Scientific reports 7: 41605.

12. Yao, Y., W. Miao, Z. Liu, W. Han, K. Shi, Y. Shen, H. Li, Q. Liu, Y. Fu, D. Huang, and F. Shi. 2016. Dimethyl Fumarate and Monomethyl Fumarate Promote Post-Ischemic Recovery in Mice. Translational stroke research 7: 535-547.

13. Ha, C., S. Park, Y. Choi, J. Jeong, C. Oh, K. Bae, S. Lee, J. Kim, K. Park, Jun, dY, and I. Lee. 2014. Activation of Nrf2 by dimethyl fumarate improves vascular calcification. Vascular pharmacology 63 : 29-36.

14. Liu, X., W. Zhou, X. Zhang, P. Lu, Q. Du, L. Tao, Y. Ding, and Y. Wang, and Hu R. 2016. Dimethyl fumarate ameliorates dextran sulfate sodium-induced murine experimental colitis by activating Nrf2 and suppressing NLRP3 inflammasome activation. Biochemical pharmacology 112: 37-49. 
15. Dwivedi, D., and G. Jena, and Kumar V. 2020. Dimethyl fumarate protects thioacetamide-induced liver damage in rats: Studies on Nrf2, NLRP3, and NF-KB. Journal of biochemical and molecular toxicology 34: e22476.

16. Amin, F., R. Abdelaziz, M. Hamed, M. Nader, and G. Shehatou. 2020. Dimethyl fumarate ameliorates diabetes-associated vascular complications through ROS-TXNIP-NLRP3 inflammasome pathway. Life sciences 256: 117887.

17. Zhang, Y., X. Li, J. Grailer, N. Wang, M. Wang, J. Yao, R. Zhong, G. Gao, P. Ward, D. Tan, and X. Li. 2016. Melatonin alleviates acute lung injury through inhibiting the NLRP3 inflammasome. Journal of pineal research 60: 405-414.

18. Jia, L., H. Hao, C. Wang, and J. Wei. 2021. Etomidate attenuates hyperoxia-induced acute lung injury in mice by modulating the Nrf2/HO-1 signaling pathway. Experimental and therapeutic medicine 22: 785.

19. Lu, Z., H. Yang, H. Cao, C. Huo, Y. Chen, D. Liu, P. Xie, H. Zhou, J. Liu, and L. Yu. 2020. Forsythoside A protects against lipopolysaccharide-induced acute lung injury through up-regulating microRNA-124. Clinical science 134: 2549-2563.

20. Fleetwood, A., M. Lee, W. Singleton, A. Achuthan, M. Lee, N. O'Brien-Simpson, A. Cook, A. Murphy, S. Dashper, E. Reynolds, and J. Hamilton. 2017. Porphyromonas gingivalisMetabolic Remodeling, Inflammasome Activation, and Pyroptosis in Macrophages Stimulated by and Its Outer Membrane Vesicles. Frontiers in cellular and infection microbiology 7: 351.

21. Tsai, C., Y. Lin, H. Wang, and T. Chou. 2014. Baicalein, an active component of Scutellaria baicalensis, protects against lipopolysaccharide-induced acute lung injury in rats. Journal of ethnopharmacology 153: 197-206.

22. Grommes, J., and O. Soehnlein. 2011. Contribution of neutrophils to acute lung injury. Molecular medicine 17: 293-307.

23. Cattani-Cavalieri, I., H. da Maia Valença, J. Moraes, L. Brito-Gitirana, B. Romana-Souza, M. Schmidt, and S. Valença. 2020. Dimethyl Fumarate Attenuates Lung Inflammation and Oxidative Stress Induced by Chronic Exposure to Diesel Exhaust Particles in Mice. International journal of molecular sciences 21: 9658.

24. Wu, D., H. Zhang, Q. Wu, F. Li, Y. Wang, S. Liu, and J. Wang. 2021. Sestrin 2 protects against LPSinduced acute lung injury by inducing mitophagy in alveolar macrophages. Life sciences 267: 118941.

25. Wang, Y., Q. Liu, Q. Zheng, T. Liu, X. Xu, X. Liu, W. Gao, X. Bai, and Z. Li. 2019. Dihydromyricetin Alleviates Sepsis-Induced Acute Lung Injury through Inhibiting NLRP3 Inflammasome-Dependent Pyroptosis in Mice Model. Inflammation 42: 1301-1310.

26. Teng, J., Q. Mei, X. Zhou, Y. Tang, R. Xiong, W. Qiu, R. Pan, B. Law, and V. Wong, and Yu C. 2020. Polyphyllin VI Induces Caspase-1-Mediated Pyroptosis via the Induction of ROS/NFKB/NLRP3/GSDMD Signal Axis in Non-Small Cell Lung Cancer. Cancers 12: 193. 
27. Wang, R., Y. Wang, L. Hu, Z. Lu, and X. Wang. 2021. Inhibition of complement C5a receptor protects lung cells and tissues against lipopolysaccharide-induced injury via blocking pyroptosis. Aging (Albany NY) 13: 8588-8598.

28. Zhang, Y., Z. Han, A. Jiang, D. Wu, S. Li, Z. Liu, Z. Wei, Z. Yang, and C. Guo. 2020. PterostilbeneProtective Effects of on Lipopolysaccharide-Induced Acute Lung Injury in Mice by Inhibiting NF-kB and Activating Nrf2/HO-1 Signaling Pathways. Frontiers in pharmacology 11: 591836.

29. Watanabe, R., H. Nakamura, H. Masutani, and J. Yodoi. 2010. Anti-oxidative, anti-cancer and antiinflammatory actions by thioredoxin 1 and thioredoxin-binding protein-2. Pharmacology \& therapeutics 127: 261-270.

30. Jia, Y., R. Cui, C. Wang, Y. Feng, Z. Li, Y. Tong, K. Qu, C. Liu, and J. Zhang. 2020. Metformin protects against intestinal ischemia-reperfusion injury and cell pyroptosis via TXNIP-NLRP3-GSDMD pathway. Redox biology 32: 101534.

31. Cen, M., W. Ouyang, W. Zhang, L. Yang, X. Lin, M. Dai, H. Hu, H. Tang, H. Liu, J. Xia, and F. Xu. 2021. MitoQ protects against hyperpermeability of endothelium barrier in acute lung injury via a Nrf2dependent mechanism. Redox biology 41: 101936.

\section{Figures}


A<smiles>COC(=O)/C=C/C(=O)OC</smiles>

C

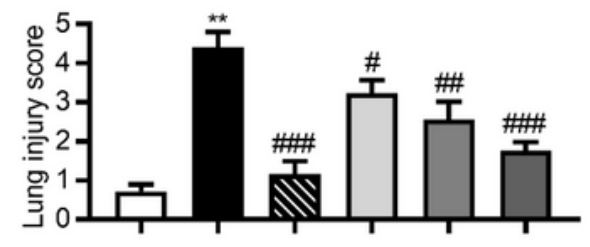

$\mathrm{E}$

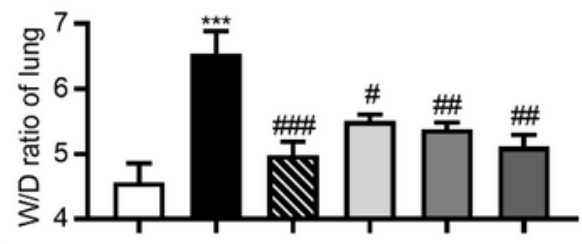

눙

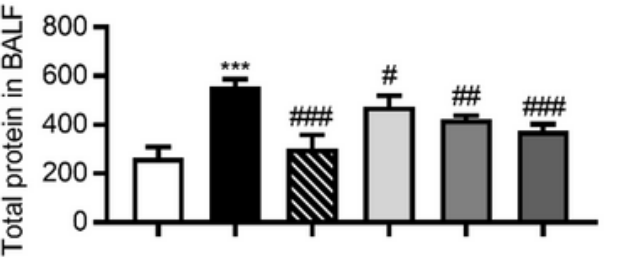

LPS $(10 \mathrm{mg} / \mathrm{kg})$ -

DXMS (50 ug/kg)

G

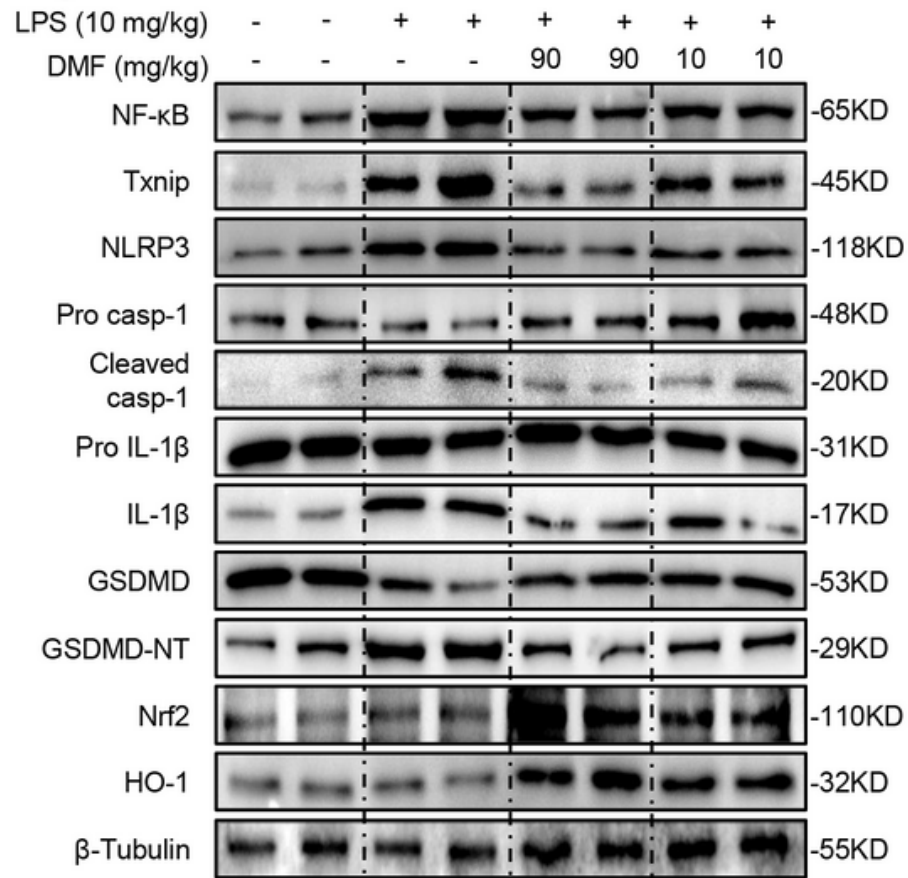

B

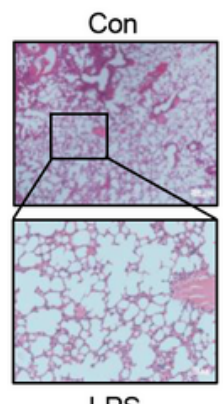

LPS
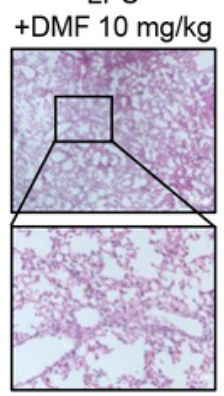

F

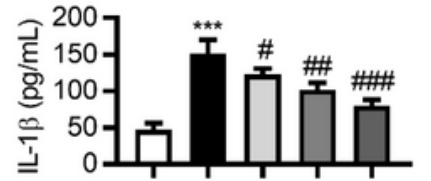

LPS (10 mg/kg)

DMF $(\mathrm{mg} / \mathrm{kg})-\quad-1030$ - 30

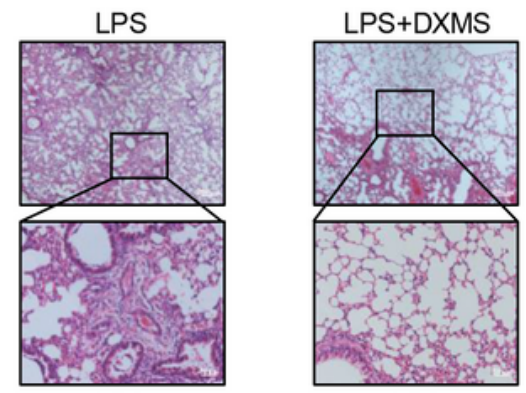

LPS

+DMF 30 mg/kg

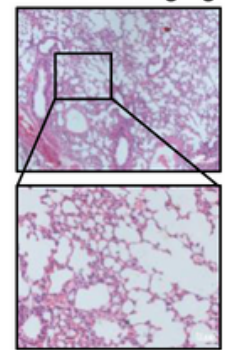

+ DMF $90 \mathrm{mg} / \mathrm{kg}$
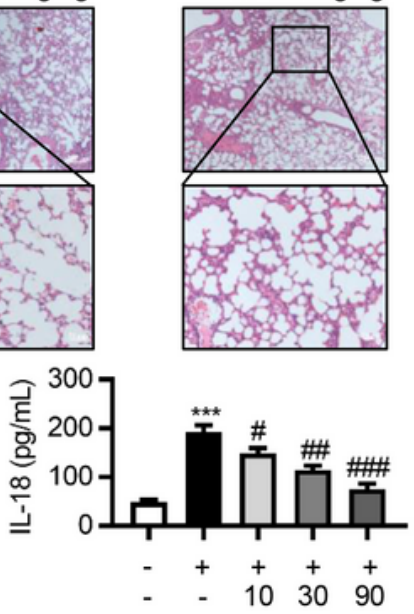
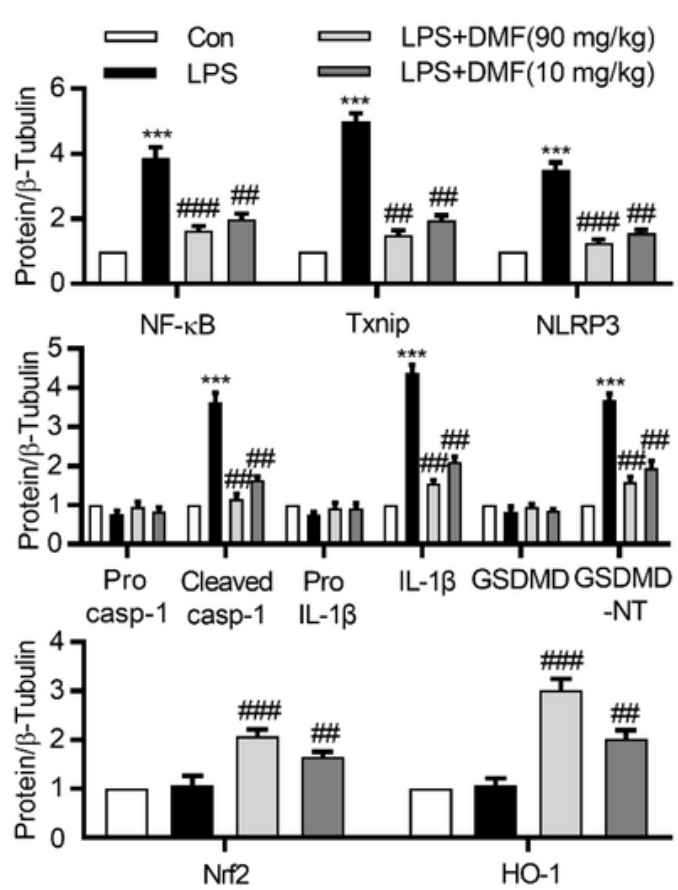

\section{Figure 1}

Protective effects of DMF treatment against LPS-induced ALI mice. a The chemical structure of DMF. $b$ Lungs from each experimental group were processed for H\&E ( $n=5$, Scale bar: $200 \mu \mathrm{m}$ and $50 \mu \mathrm{m})$. c The lung injury score was determined following a four-point scale from 0 to 3 as follows: $0,1,2$ and 3 represent no damage, mild damage, moderate damage and severe damage, respectively. $d$ The lung W/D ratio was determined. e BALF was collected to measure amount of protein. $f$ The serum levels of IL-1 $\beta$ 
and IL-18 were determined by enzyme-linked immunosorbent assay (ELISA). g The expressions of NLRP3, NF-kB, Txnip, Nrf2 and HO-1, and the cleavage of casapase-1, IL-1 $\beta$, GSDMD were measured by Western blot analysis, and $\beta$-Tubulin was acted as an internal control. Data are presented as mean \pm SD. ${ }^{*} p<$ 0.05 , $* * p<0.005$ and $* * * p<0.001$ vs Control group; $\# p<0.05$, \#\# $p<0.005$ and \#\#\#p<0.001 vs LPS group.

A

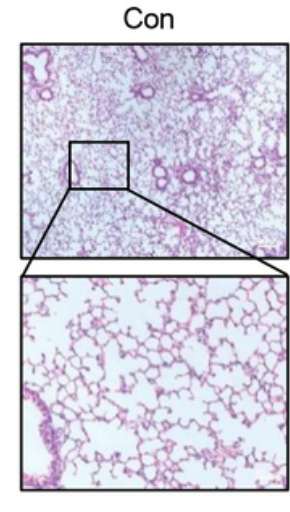

B

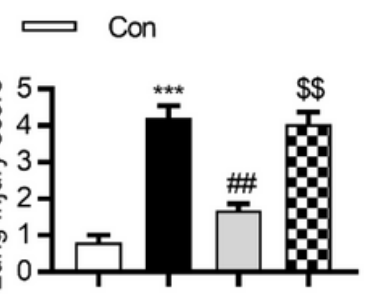

LPS

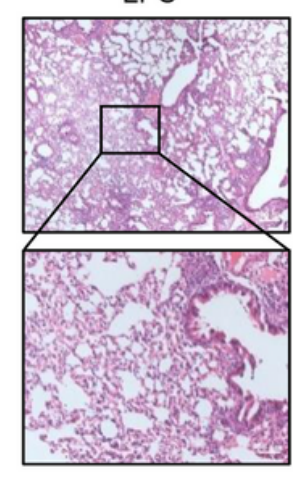

C

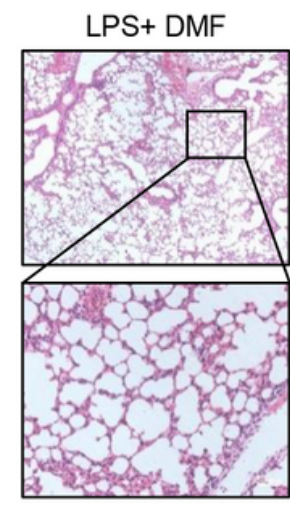

ए LPS+DMF

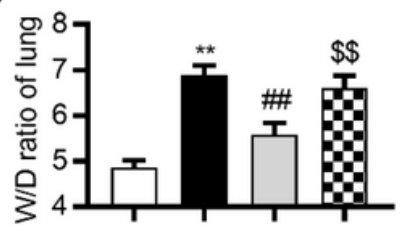

$\mathrm{D}$

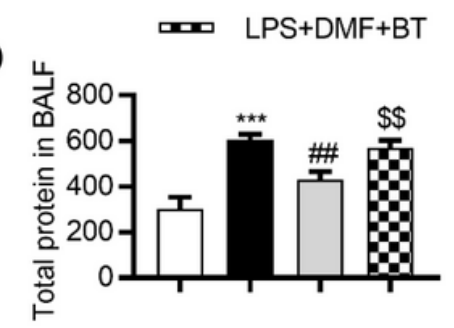

E
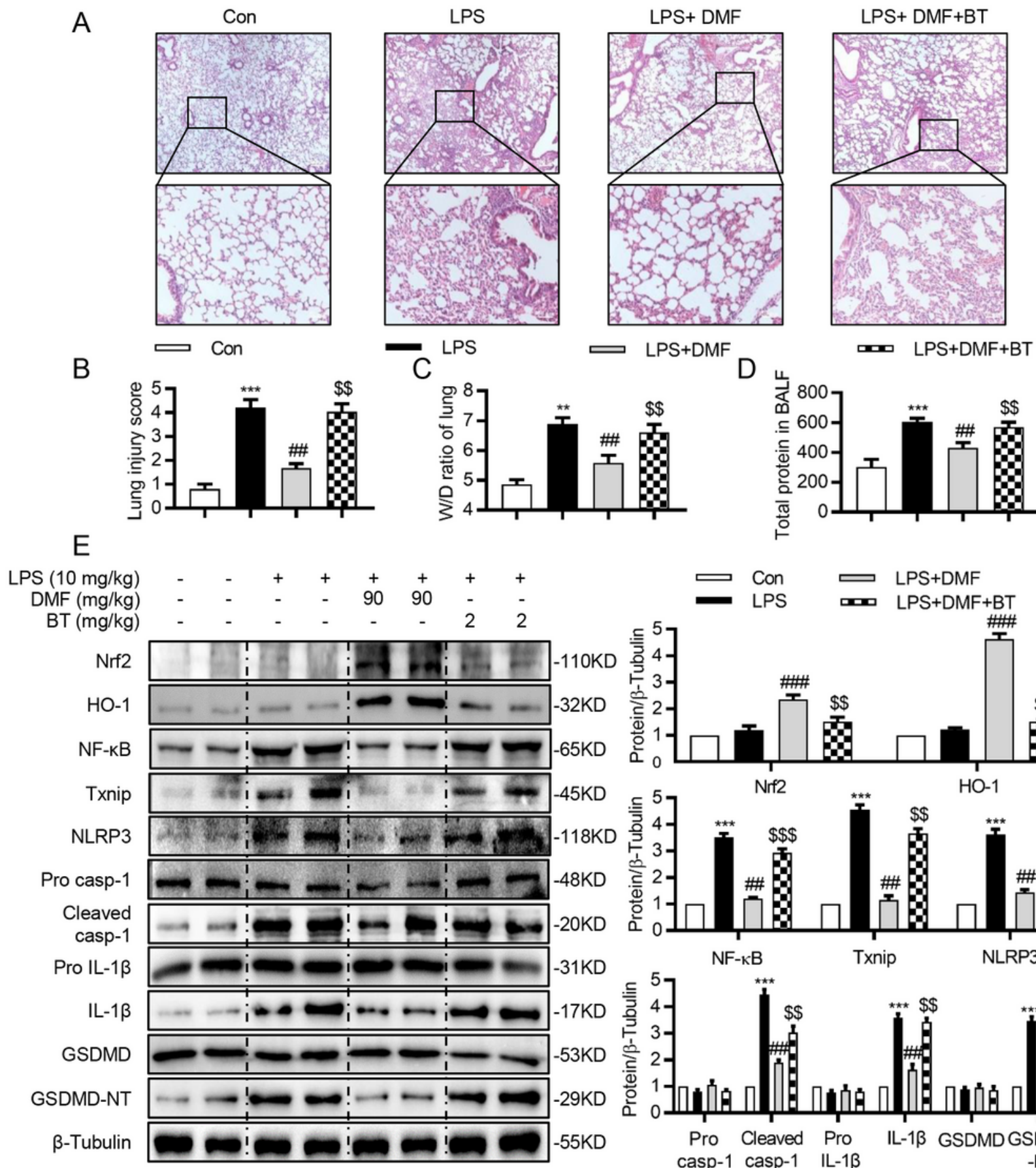

LPS+DMF+BT
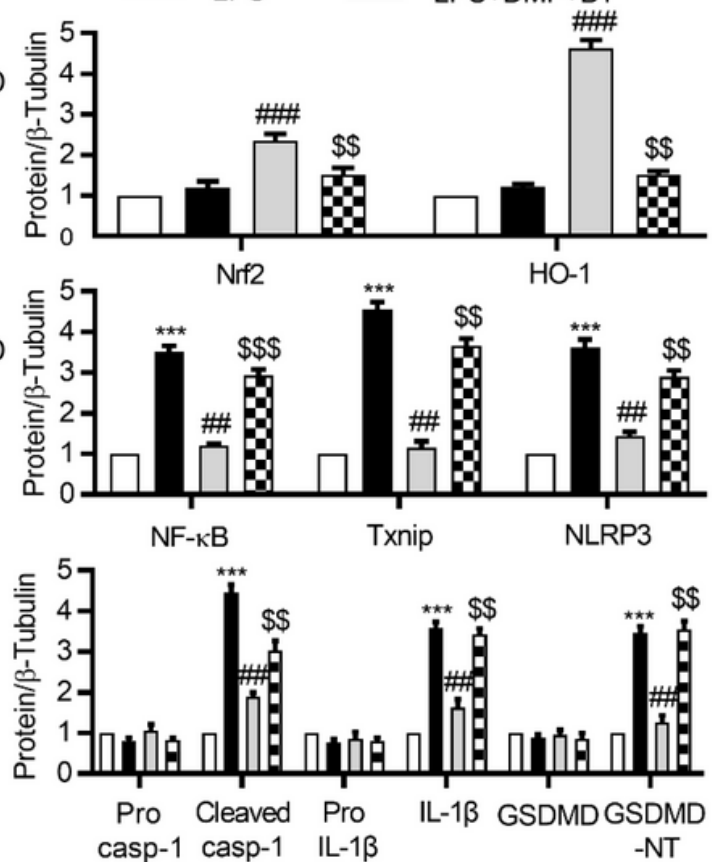

Figure 2

Nrf2 inhibitor reduced the beneficial effects of DMF in LPS-induced ALI mice. a Representative histological sections of the lungs were stained with $\mathrm{H} \& E(n=5$, Scale bar: $200 \mu \mathrm{m}$ and $50 \mu \mathrm{m})$. b The lung injury score was determined. $c$ The lung W/D ratio was determined. $d$ BALF was collected to measure 
amount of protein. e The expressions of Nrf2, HO-1, NF-KB, Txnip, NLRP3, and the cleavage of casapase1 , IL-1 $\beta$, GSDMD were measured by Western blot analysis, and $\beta$-Tubulin was acted as an internal control. Data are presented as mean \pm SD. ${ }^{* \star} p<0.005$ and ${ }^{* \star *} p<0.001$ vs Control group; \#\# $p<0.005$ and \#\#\# $p<0.001$ vs LPS group; $\$ p<0.005$ vs DMF group.
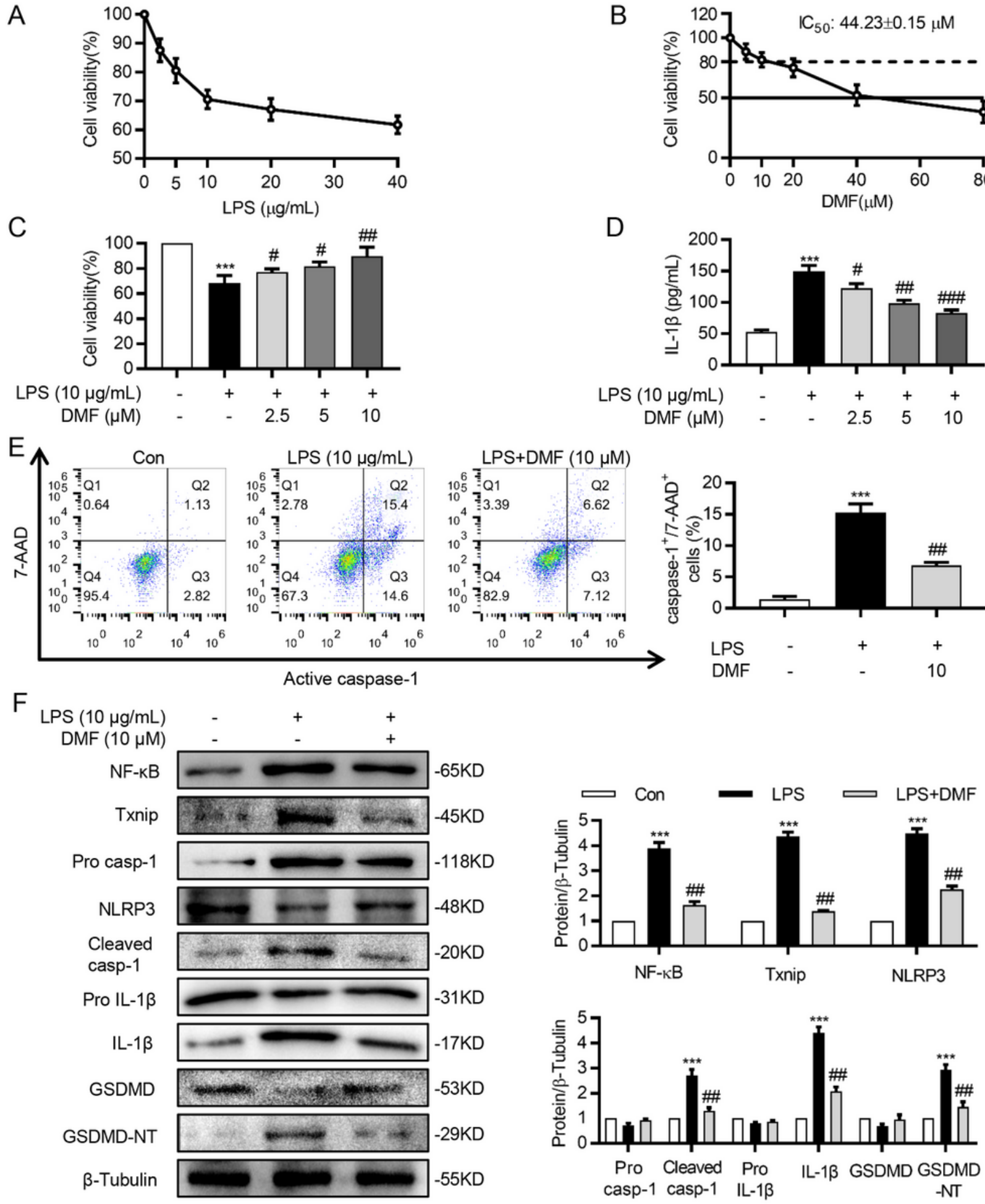

Figure 3

Effects of DMF exposure on LPS-induced NLRP3 inflammasome and pyroptosis in BEAS-2B cells. Cell viability after LPS a or DMF b exposure was measured by an MTT assay. c Cell viability after LPS 
exposure was measured by MTT assay. Cells were subjected to $\operatorname{DMF}(2.5,5$, or $10 \mu \mathrm{M})$ and DXMS (100 $\mathrm{nM})$, and then exposed to LPS $(10 \mu \mathrm{g} / \mathrm{mL})$ for $24 \mathrm{~h}$. $\mathrm{d}$ Levels of IL-1 $\beta$ in culture supernatants were measured by ELISA. e 7-AAD (vertical) and active caspase-1 (horizontal) measured by flow cytometry. $f$ The protein expression of NF-KB, Txnip, NLRP3, casapase-1, IL-1 $\beta$, and GSDMD was measured by Western blot analysis, and $\beta$-Tubulin was acted as an internal control. All data are presented as means $\pm S D(n=3$ in each group). ${ }^{* * *} p<0.001$ vs Control group; $\# p<0.05$, \#\# $p<0.005$ and $\# \# \# p<0.001$ vs LPS group.

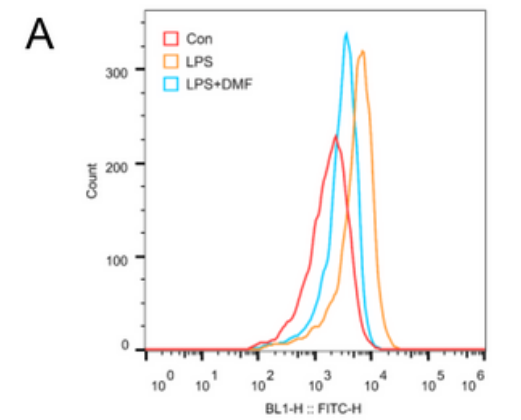

C

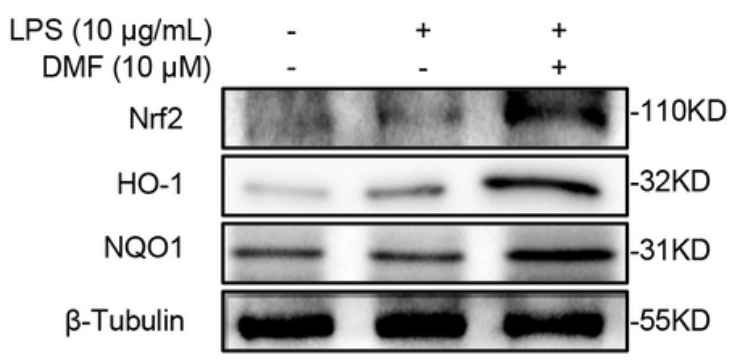

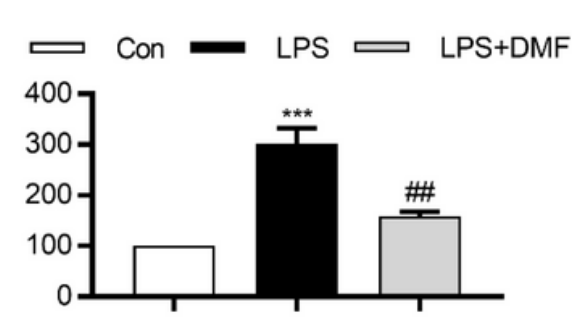

$\mathrm{B}$

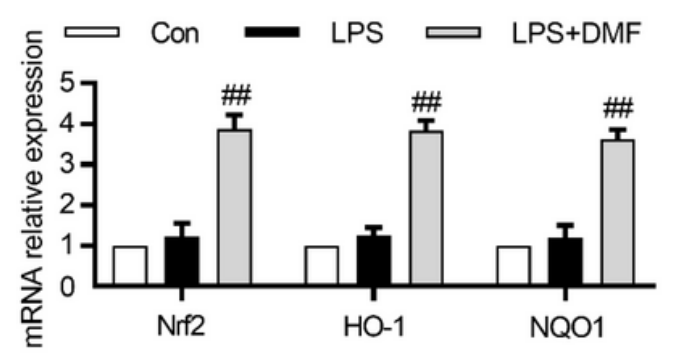

\section{Figure 4}

Effects of DMF exposure on LPS-induced ROS generation in BEAS-2B cells. a ROS generation was determined using flow cytometry in BEAS-2B cells. $b$ The mRNA expression of Nrf2 and its target gene HO-1, NQ01 was analyzed by Real-time RT-PCR. c The protein expression of Nrf2 and HO-1 was measured by Western blot analysis, and $\beta$-Tubulin was acted as an internal control. All data are presented as means $\pm S D$ ( $n=3$ in each group). ${ }^{* *} p<0.001$ vs Control group; $\# \# p<0.005$ and $\# \# \# p<0.001$ vs LPS group. 

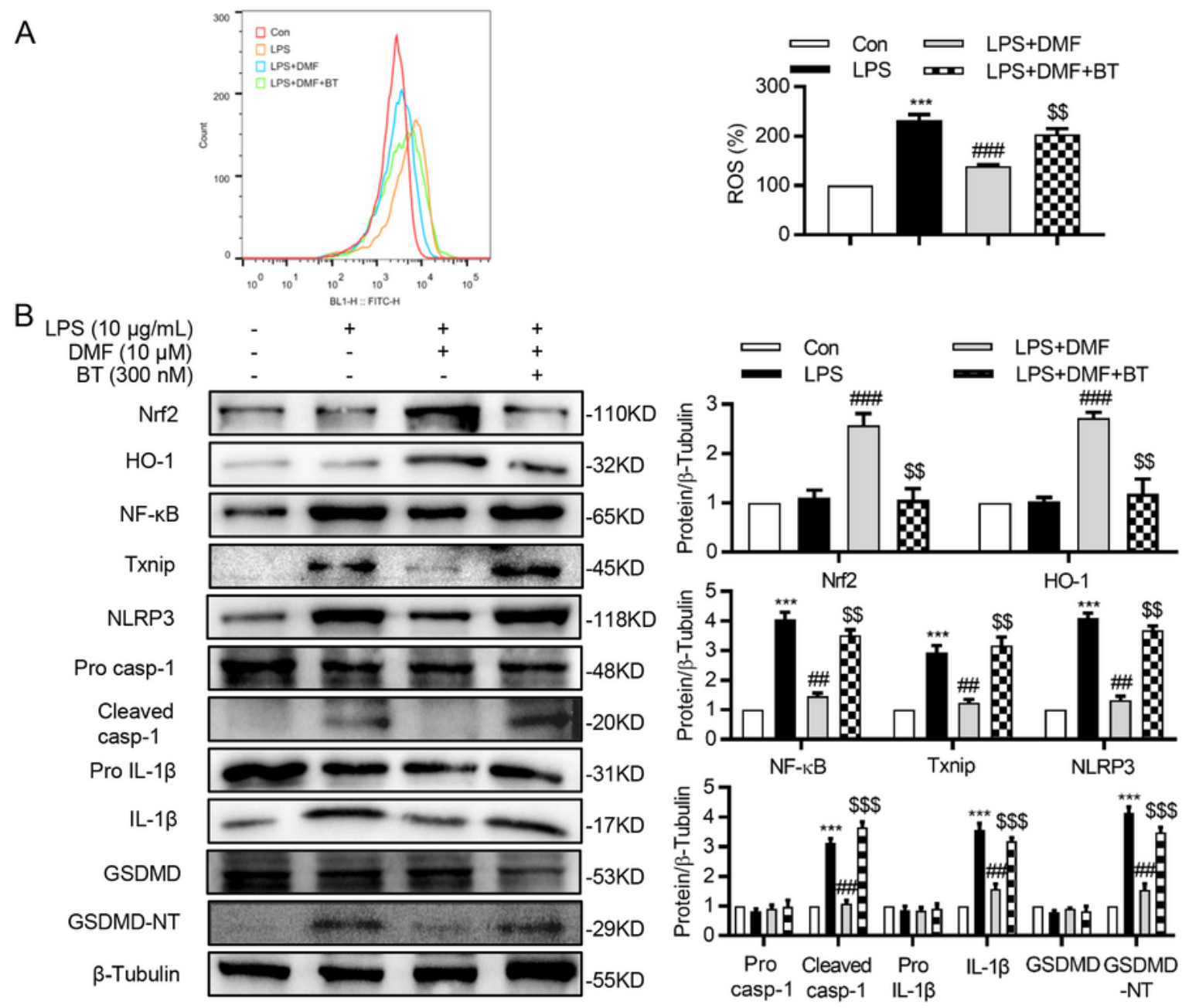

C

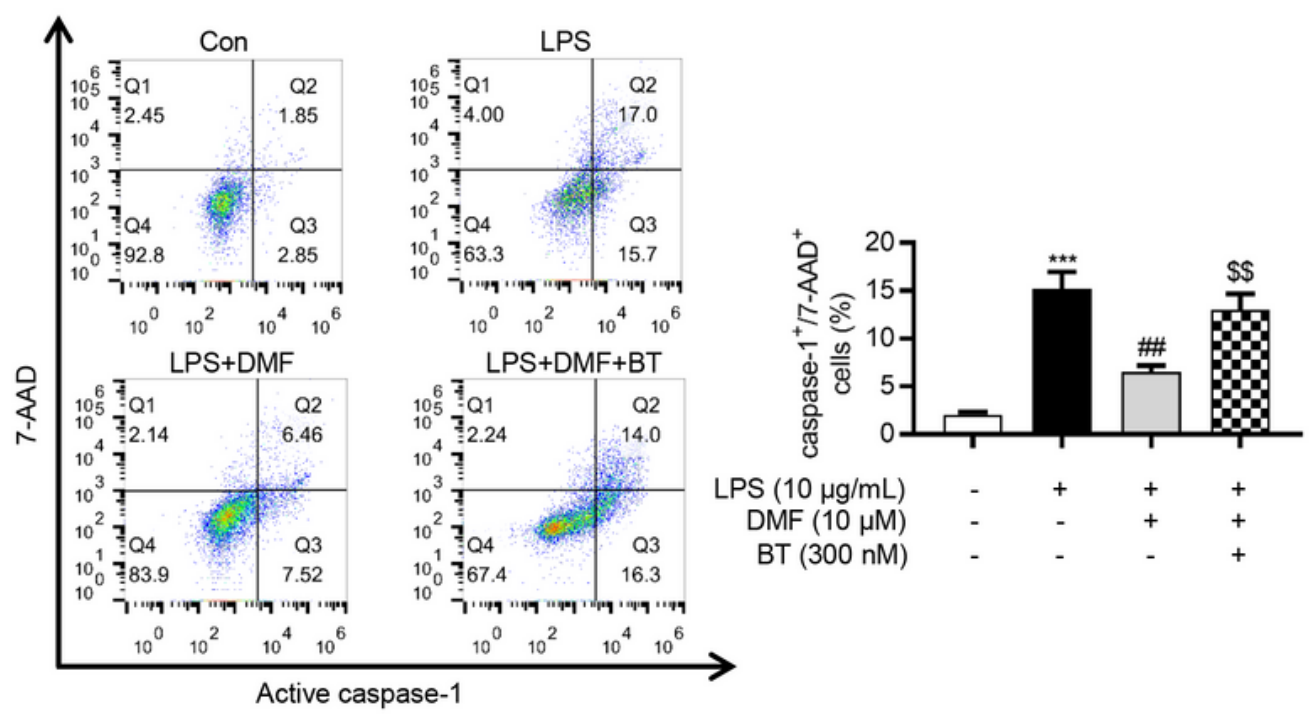

\section{Figure 5}

Effects of Nrf2 inhibitor on LPS-stimulated BEAS-2B cells. a ROS generation was determined using flow cytometry in BEAS-2B cells. b The expression of Nrf2, HO-1, NF-KB, Txnip, NLRP3, and the cleavage of casapase-1, IL-1 $\beta$, GSDMD were measured by Western blot analysis, and $\beta$-Tubulin was acted as an internal control. c Double positive staining 7-AAD (vertical) and active caspase-1 cells (horizontal) were 
measured by flow cytometry. All data are presented as means $\pm S D\left(n=3\right.$ in each group). ${ }^{\star \star \star} p<0.001$ vs Control group; \#\# $p<0.005$ and \#\#\# $p<0.001$ vs LPS group;

$$
p<0.005 \text { and }
$$

$\$ p<0.005$ vs DMF group.

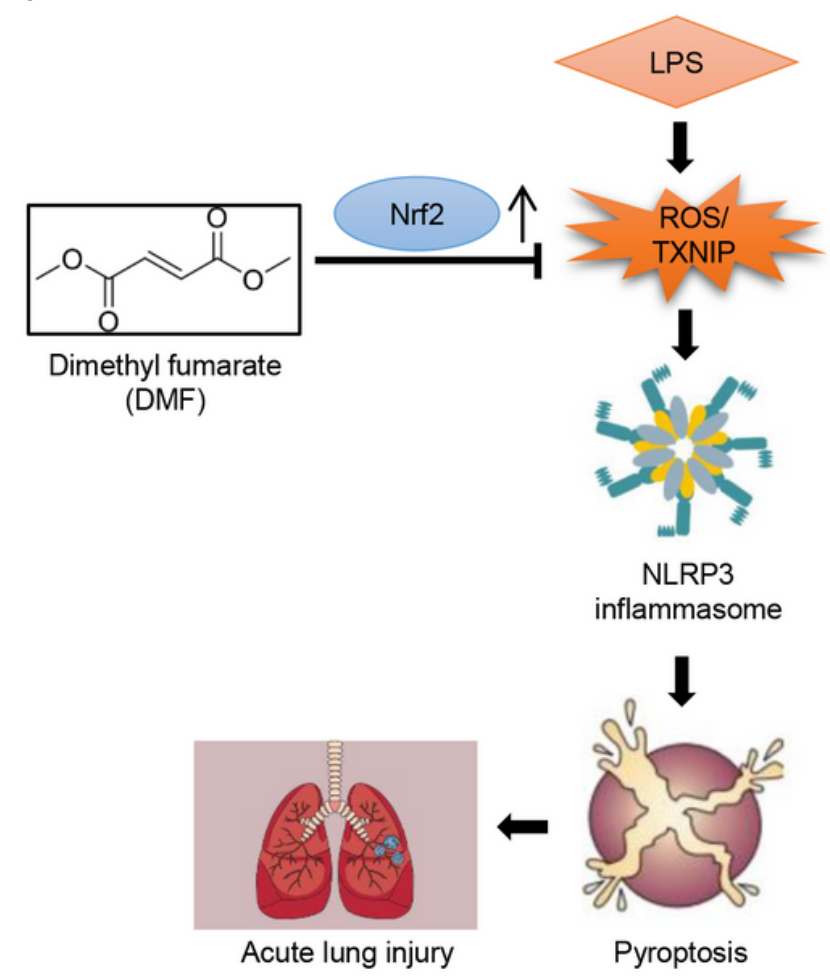

\section{Figure 6}

DMF could improve LPS-induced acute lung injury via inhibiting NLPR3 inflammasome and related pyroptosis, and that these effects were mediated by triggering Nrf2 expression. 